\title{
APPROXIMATE PROPER VECTORS
}

\author{
STERLING K. BERBERIAN
}

1. Notations and terminology. Our terminology conforms with that of [2]. The inner product of vectors $x$ and $y$ in a Hilbert space $\mathfrak{H C}$ is denoted $(x, y)$. An operator in $\mathcal{H}$ is a continuous linear mapping $T: \mathfrak{H C} \rightarrow \mathfrak{H C}$. The *-algebra of all operators in $\mathfrak{H C}$ is denoted $L(\mathfrak{H C})$. A complex number $\mu$ is a proper value for $T$ if there exists a nonzero vector $x$ such that $(T-\mu I) x=0$; such a vector $x$ is a proper vector for $T$. A complex number $\mu$ is an approximate proper value for $T$ in case there exists a sequence of vectors $x_{n}$ such that $\left\|x_{n}\right\|=1$ and $\left\|T x_{n}-\mu x_{n}\right\|$ $\rightarrow 0$; equivalently, there does not exist a number $\epsilon>0$ such that $(T-\mu I)^{*}(T-\mu I) \geqq \epsilon I$.

The spectrum of an operator $T$, denoted $s(T)$, is the set of all complex numbers $\mu$ such that $T-\mu I$ has no inverse. The approximate point spectrum of $T$, denoted $a(T)$, is the set of all approximate proper values of $T$. The point spectrum of $T$, denoted $p(T)$, is the set of all proper values of $T$. Evidently $p(T) \subset a(T) \subset s(T)$. If $T$ is normal, $s(T)=a(T)$ (see [2, Theorem 31.2]); if $T$ is Hermitian, $a(T)$ contains a (necessarily real) number $\alpha$ such that $|\alpha|=\|T\|$ (see [2, Theorem $34.2])$, and in particular one has an elementary proof of the fact that the spectrum of $T$ is nonempty.

2. Introduction. The spectrum of a Hermitian operator is shown to be nonempty by completely elementary means. It would be nice to have an elementary proof for normal operators (see [2, p. 111]). The purpose of this note is to give a proof based on Banach limits. Incidentally, $\mathfrak{H}$ will be extended to a curious Hilbert space $\mathcal{K}$, in which it becomes natural to speak of "approximate proper vectors."

Our motivation for the construction of $\mathfrak{K}$ was as follows. Suppose $T$ is a normal operator, and $\mu$ and $\nu$ are distinct approximate proper values of $T$. Choose sequences of unit vectors $\left\{x_{n}\right\}$ and $\left\{y_{n}\right\}$ such that $\left\|T x_{n}-\mu x_{n}\right\| \rightarrow 0$ and $\left\|T y_{n}-\nu y_{n}\right\| \rightarrow 0$. Then,

$$
\begin{aligned}
\left|(\mu-\nu)\left(x_{n}, y_{n}\right)\right| & =\left|\left(\mu x_{n}-T x_{n}, y_{n}\right)+\left(x_{n}, T^{*} y_{n}-\nu^{*} y_{n}\right)\right| \\
& \leqq\left\|\mu x_{n}-T x_{n}\right\|+\left\|T^{*} y_{n}-\nu^{*} y_{n}\right\| \\
& =\left\|\mu x_{n}-T x_{n}\right\|+\left\|T y_{n}-\nu y_{n}\right\| \rightarrow 0 .
\end{aligned}
$$

Thus, $\left(x_{n}, y_{n}\right) \rightarrow 0$, and we have a generalization of the following well-

Presented to the Society, April, 14, 1961; received by the editors December 21, 1960 and, in revised form, January 20, 1961. 
known fact: for a normal operator, proper vectors belonging to distinct proper values are orthogonal. This suggests thinking of the sequences $\left\{x_{n}\right\}$ and $\left\{y_{n}\right\}$ as being "approximate proper vectors," with their inner product defined to be $\lim \left(x_{n}, y_{n}\right)$.

In what follows, we denote by glim a fixed "Banach generalized limit," defined for bounded sequences $\left\{\lambda_{n}\right\}$ of complex numbers (see page 34 of $[1])$; thus,

$$
\begin{aligned}
\operatorname{glim}\left(\lambda_{n}+\mu_{n}\right) & =\operatorname{glim} \lambda_{n}+\operatorname{glim} \mu_{n}, \\
\operatorname{glim}\left(\lambda \lambda_{n}\right) & =\lambda \operatorname{glim} \lambda_{n}, \\
\operatorname{glim} \lambda_{n} & =\lim \lambda_{n} \text { whenever }\left\{\lambda_{n}\right\} \text { is convergent, } \\
\operatorname{glim} \lambda_{n} & \geqq 0 \text { when } \lambda_{n} \geqq 0 \text { for all } n .
\end{aligned}
$$

We shall not make use of a "translation-invariant" property of glim; all we need are properties (1)-(4), in other words, a positive linear form on the vector space $m$ of bounded sequences, which vanishes on the space $c_{0}$ of null sequences, and has the value 1 for the constant sequence $\{1\}$. It follows from (1) and (4) that glim $\lambda_{n}$ is real whenever $\lambda_{n}$ is real for all $n$; this implies in turn that $\operatorname{glim}\left(\lambda_{n}^{*}\right)=\left(\operatorname{glim} \lambda_{n}\right)^{*}$ for any bounded sequence $\left\{\lambda_{n}\right\}$.

3. An extension $\mathscr{K}$ of $\mathcal{H}$. Denote by $B$ the set of all sequences $s=\left\{x_{n}\right\}$, with $x_{n}$ in $\mathcal{H C}(n=1,2,3, \cdots)$, such that $\left\|x_{n}\right\|$ is bounded [that is, $\left\{\left\|x_{n}\right\|\right\}$ is in $m$ ]. If $s=\left\{x_{n}\right\}$ and $t=\left\{y_{n}\right\}$, write $s=t$ in case $x_{n}=y_{n}$ for all $n$. The set $B$ is a vector space relative to the definitions $s+t=\left\{x_{n}+y_{n}\right\}$ and $\lambda s=\left\{\lambda x_{n}\right\}$.

Suppose $s=\left\{x_{n}\right\}$ and $t=\left\{y_{n}\right\}$ belong to $B$; since $\left|\left(x_{n}, y_{n}\right)\right|$ $\leqq\left\|x_{n}\right\|\left\|y_{n}\right\|$, it is permissible to define

$$
\phi(s, t)=\operatorname{glim}\left(x_{n}, y_{n}\right) .
$$

Evidently $\phi$ is a positive symmetric bilinear functional on $B$ (see $[2, \S 2])$, hence $|\phi(s, t)|^{2} \leqq \phi(s, s) \phi(t, t)$ (see $\left.[2, \S 5]\right)$. Let $\mathfrak{N}=\{s: \phi(s, s)=0\}=\{s: \phi(s, t)=0$ for all $t$ in $B\}$. Clearly $\mathscr{N}$ is a linear subspace of $B$; we write $s^{\prime}$ for the coset $s+\Re$. The quotient vector space $P=B / \Re$ becomes an inner product space on defining $\left(s^{\prime}, t^{\prime}\right)=\phi(s, t)$. Thus, if $u=\left\{x_{n}\right\}^{\prime}$ and $v=\left\{y_{n}\right\}^{\prime}$,

$$
(u, v)=\operatorname{glim}\left(x_{n}, y_{n}\right) \text {. }
$$

If $x$ is in $\mathcal{H}$, we write $\{x\}$ for the sequence all of whose terms are $x$, and $x^{\prime}$ for the coset $\{x\}+\mathscr{x}$. Evidently $\left(x^{\prime}, y^{\prime}\right)=(x, y)$, and $x \rightarrow x^{\prime}$ is an isometric linear mapping of $\mathfrak{H C}$ onto a closed linear subspace ${ }^{\prime} C^{\prime}$ of $P$. Regard $P$ as a linear subspace of its Hilbert space completion 
$\mathcal{K}$. Thus, $\mathcal{H}^{\prime} \subset \mathcal{P} \subset \mathcal{K}$, where $\mathcal{H}^{\prime}$ is a closed linear subspace of $\mathcal{K}$, and $P$ is a dense linear subspace of $\mathscr{K}$.

4. A representation of $L(\mathcal{H C})$. Every operator $T$ in $\mathcal{H C}$ determines an operator $T^{0}$ in $\mathcal{K}$, as follows.

If $s=\left\{x_{n}\right\}$ is in $B$, then the relation $\left\|T x_{n}\right\|^{2} \leqq\|T\|\left\|^{2}\right\| x_{n} \|^{2}$ shows that $\left\{T x_{n}\right\}$ is in $B$. Defining $T_{0} s=\left\{T x_{n}\right\}$, we have a linear mapping $T_{0}: B \rightarrow B$ such that $\phi\left(T_{0} s, T_{0} s\right) \leqq\|T\|^{2} \phi(s, s)$. In particular if $s$ is in $\Re$, that is if $\phi(s, s)=0$, then $T_{0} s$ is also in $\mathscr{~}$. It follows that $\left\{x_{n}\right\}^{\prime}$ $\rightarrow\left\{T x_{n}\right\}^{\prime}$ is a well-defined linear mapping of $P$ into $P$, which we denote $T^{0}$; thus, $T^{0} s^{\prime}=\left(T_{0} s\right)^{\prime}$, and the inequality $\left(T^{0} u, T^{0} u\right) \leqq\|T\|^{2}(u, u)$, valid for all $u$ in $\odot$, shows that $T^{0}$ is continuous, with $\left\|T^{0}\right\| \leqq\|T\|$. Since in particular $T^{0} x^{\prime}=(T x)^{\prime}$ for all $x$ in $\mathcal{F}$, it is clear that $\left\|T^{0}\right\|$ $\geqq\|T\|$, thus $\left\|T^{0}\right\|=\|T\|$. The continuous linear mapping $T^{0}$ extends to a unique operator in $\mathscr{K}$, which we also denote $T^{0}$.

The mapping $T \rightarrow T^{0}$ of $L(\mathfrak{K C})$ into $L(\mathfrak{K})$ is easily seen to be a faithful *-representation: $(S+T)^{0}=S^{0}+T^{0},(\lambda T)^{0}=\lambda T^{0},(S T)^{0}=S^{0} T^{0}$, $\left(T^{*}\right)^{0}=\left(T^{0}\right)^{*}, I^{0}=I$, and $\left\|T^{0}\right\|=\|T\|$.

Suppose $T \geqq 0$, that is, $(T x, x) \geqq 0$ for all $x$ in $\nVdash$. If $u=\left\{x_{n}\right\}^{\prime}$ is in $\odot$, then $\left(T x_{n}, x_{n}\right) \geqq 0$ for all $n$, hence $\left(T^{0} u, u\right)=\operatorname{glim}\left(T x_{n}, x_{n}\right) \geqq 0$; it follows that $\left(T^{0} v, v\right) \geqq 0$ for all $v$ in $\mathfrak{K}$. Clearly: for an operator $T$ in $\mathfrak{H C}$, one has $T \geqq 0$ if and only if $T^{0} \geqq 0$.

LEMMA. If $T$ is any operator in $\mathfrak{F}, a\left(T^{0}\right)=a(T)$.

Proof. A complex number $\mu$ fails to belong to $a(T)$ if and only if there exists a number $\epsilon>0$ such that $(T-\mu I)^{*}(T-\mu I) \geqq \epsilon I$. By the above remarks, this condition is equivalent to $\left(T^{0}-\mu I\right)^{*}\left(T^{0}-\mu I\right)$ $\geqq \epsilon I$.

TheOREM 1. For every operator $T$ in $\mathfrak{H}$,

$$
a(T)=a\left(T^{0}\right)=p\left(T^{0}\right) .
$$

Proof. The relations $a(T)=a\left(T^{0}\right) \supset p\left(T^{0}\right)$ have already been noted. Suppose $\mu$ is in $a(T)$. Choose a sequence $x_{n}$ in $\mathcal{H C}$ such that $\left\|x_{n}\right\|=1$ and $\left\|T x_{n}-\mu x_{n}\right\| \rightarrow 0$, and set $u=\left\{x_{n}\right\}^{\prime}$. Clearly $\|u\|=1$ and $\left\|T^{0} u-\mu u\right\|^{2}$ $=\operatorname{glim}\left\|T x_{n}-\mu x_{n}\right\|^{2}=0$, hence $T^{0} u=\mu u$; that is, $\mu$ is in $p\left(T^{0}\right)$.

THEOREM 2. If $T$ is any normal operator in $\mathfrak{H}, T$ has an approximate proper value $\mu$ such that $|\mu|=\|T\|$.

Proof. Without loss of generality, we may suppose $\|T\|=1$. If 1 is in $s(T)$, the relation $s(T)=a(T)$ ends the proof. Let us assume henceforth that $I-T$ is invertible. 
Let $S=T^{*} T$. Since $\|S\|=1$, and since $S \geqq 0$, it follows from the remarks in $\$ 1$ that 1 is an approximate proper value for $S$. By Theorem 1,1 is a proper value for $S^{0}$. Let $\mathfrak{T}$ be the null space of $S^{0}-I$, thus $\mathfrak{T l}=\left\{v: S^{0} v=v\right\} \neq\{0\}$. Since $T S=S T$ and $T^{*} S=S T^{*}, \mathfrak{T}$ is invariant under $T^{0}$ and $\left(T^{0}\right)^{*}$; thus, $\mathscr{T}$ reduces $T^{0}$. We denote by $T^{0} / \mathscr{T} C$ the restriction of $T^{0}$ to $\mathfrak{T}$. Since $S^{0} / \mathfrak{T} C=I$, we have $\left(T^{0} / \mathfrak{T} \mathcal{C}\right)^{*}\left(T^{0} / \mathfrak{T} \mathrm{C}\right)$ $=\left(T^{0 *} / \mathfrak{T C}\right)\left(T^{0} / \mathfrak{T}\right)=\left(T^{0 *} T^{0}\right) / \mathfrak{T C}=S^{0} / \mathfrak{T C}=I ;$ clearly $T^{0} / \mathfrak{T}$ is a unitary operator in $\mathfrak{T}$. Write $U=T^{0} / \mathfrak{T}$. Since $I-T$ has an inverse in $L(\mathcal{H C}), I-T^{0}$ has an inverse in $L(\mathcal{K})$; since $\mathscr{T}$ reduces $I-T^{0}$, it follows that $I-U$ has an inverse in $L(\mathscr{T})$. Let $R$ be the Cayley transform of $U$, that is, $R=i(I+U)(I-U)^{-1} ; R$ is a Hermitian operator in $\mathfrak{T}$. Define $A=i(I+T)(I-T)^{-1}$; clearly $A^{0} / \mathfrak{T} C=R$.

Let $\alpha$ be any approximate proper value for $R$ (see $\$ 1$ ). It is clear from the definition that $\alpha$ is also an approximate proper value for $A^{0}$. By Theorem 1 , there is a nonzero vector $u$ in $\sigma^{\rho}$ such that $A^{0} u=\alpha u$. Since $A^{0}=i\left(I+T^{0}\right)\left(I-T^{0}\right)^{-1}$, an elementary calculation gives $T^{0} u$ $=(\alpha-i)(\alpha+i)^{-1} u$. Thus, $\mu=(\alpha-i)(\alpha+i)^{-1}$ belongs to $p\left(T^{0}\right)=a\left(T^{0}\right)$ $=a(T)$, and $|\mu|=1=\|T\|$.

\section{REFERENCES}

1. S. Banach, Thérie des operations lineaires, Warsaw, 1932.

2. P. R. Halmos, Introduction to Hilbert space and the theory of spectral multiplicity, Chelsea, New York, 1951.

State University of Iowa 